\title{
Importance of knee flexion range of motion during the acute phase after total knee arthroplasty
}

\author{
Tomohiro OKA, PT, MSc ${ }^{1,2}$, Osamu WADA, PT, MSc ${ }^{1}$, Tsuyoshi AsAI, PT, Ph.D ${ }^{3}$, \\ Hideto MARUnO, $\mathrm{MD}^{4}$ and Kiyonori MIZUNO, $\mathrm{MD}^{4}$ \\ ${ }^{1)}$ Department of Rehabilitation, Anshin Hospital \\ ${ }^{2}$ Department of Public Health, Kobe University Graduate School of Health Sciences \\ ${ }^{3)}$ Department of Medical Rehabilitation, Faculty of Rehabilitation, Kobe Gakuin University \\ ${ }^{4)}$ Department of Orthopedics, Anshin Hospital
}

\begin{abstract}
Background: We investigate the association with knee flexion range of motion (ROM) during the acute phases and that at $\mathbf{1 2}$ months after total knee arthroplasty (TKA). We also clarified the cut-off ROM during the acute phases in predicting the goal of knee flexion ROM at 12 months. Methods: In this retrospective study, 193 patients with knee osteoarthritis (female:144 patients, age:73.2 \pm 7.7 years) who underwent unilateral TKA at an orthopedic clinic were recruited. They underwent assessments of knee flexion ROM at 5 days, 1 month, and 12 months after TKA. The goal of knee flexion ROM at 12 months after TKA was set at $120^{\circ}$. Single and logistic-regression analyses were performed with the dependent variables including the outcome of the goal of knee flexion ROM at 12 months, and the independent variables included knee flexion ROM at 5 days and 1 month, separately. We calculated the cut-off ROM at 5 days and 1 month for predicting the goal of knee flexion ROM at 12 months with receiver operating curve analysis. Results: Knee flexion ROM at 5 days and 1 month were significantly associated with the goal of that at 12 months (p < 0.01). The cut-off $\mathrm{ROM}$ were $85^{\circ}$ at 5 days and $105^{\circ}$ at 1 month separately. Conclusions: Our results suggest the importance of early improvement in knee flexion ROM after TKA, and that at 1 month postoperatively indicates the likelihood of achievement of the goal of knee flexion ROM at 12 months after TKA.
\end{abstract}

Key words: knee flexion range of motion, acute phase, total knee arthroplasty

(Phys Ther Res 23: 143-148, 2020)

T otal knee arthroplasty (TKA) is a standard surgical treatment for end-stage knee osteoarthritis (OA). One of the clinical goals of TKA is to provide pain relief and to restore the range of motion (ROM) of the knee joint, which contributes significantly to the clinical outcome following $\mathrm{TKA}^{1)}$. Several studies have demonstrated that knee flexion ROM is positively associated with knee function, and greater knee flexion ROM results in better clinical outcomes following $\mathrm{TKA}^{2-6)}$. In line with this, restricted knee flexion ROM after TKA has been reported to be associated with restriction of activities of daily living, lower quality of

Received: June 14, 2019

Accepted: April 2, 2020

Advance Publication by J-STAGE: August 5, 2020

Correspondence to: Tomohiro Oka, Anshin Hospital, 1-4-12 Minatojima Minamimachi, Chuo-ku, Kobe, Hyogo 650-0047, Japan

\# e-mail: tomohiro1986640@yahoo.co.jp

doi: 10.1298/ptr.E9996 life, and patient dissatisfaction with the treatment ${ }^{1,5-7)}$. Therefore, the improvement of postoperative knee flexion ROM is clinically relevant since it aids in the achievement of successful TKA.

Knee flexion ROM after TKA has been shown to have significant correlations with age, body mass index (BMI), implant design, surgical technique, pain, and preoperative $\mathrm{ROM}^{8-13)}$. Additionally, knee flexion ROM after TKA is associated with acute postoperative $\mathrm{ROM}^{14)}$. A previous study reported that knee flexion ROM at 6 days after TKA was a predictive factor for knee flexion ROM at 2 months after $\mathrm{TKA}^{14)}$. Acute postoperative knee flexion ROM may also be predictive of knee flexion ROM at 12 months after TKA. However, the relationship between knee flexion ROM during acute postsurgical phases, and at 12 months after TKA, remains unknown. Furthermore, Ebert et al. identified a 7week postoperative cut-off point for achieving maximal knee flexion ROM after TKA ${ }^{15}$. However, the follow-up 
period in this study was insufficient because postoperative knee flexion ROM continued to improve for up to 12 months after $\mathrm{TKA}^{16)}$. Prediction of long-term knee flexion ROM based on values during the acute postoperative phases allows for better identification of patients progressing poorly who may require early intervention. Therefore, it is necessary to assess whether knee flexion ROM during the acute phases is predictive of knee flexion ROM at 12 months after TKA, in order to assist in the planning of postacute rehabilitation. The degree of knee flexion during the acute phases may be a useful clinical indicator of postoperative rehabilitation for surgeons and therapists.

The aim of the present study was to investigate whether knee flexion ROM during the acute phases, such as within 5 days and 1 month, were useful for predicting achievement of knee flexion ROM at 12 months. In addition, we identified the degree of knee flexion ROM during the acute phases that represents the cut-off point for achieving the goal of knee flexion ROM at 12 months after TKA. We hypothesized that the knee flexion ROM during the acute phases predicts the achievement of the goal of knee flexion ROM at 12 months after TKA.

\section{Methods}

\section{Participants}

In this retrospective study, 193 patients who underwent unilateral TKA at an orthopedic clinic between November 2012 and December 2015 were recruited. The participants were categorized as grade 3 or 4 based on the Kellgren and Lawrence radiographic grading system (K/L grade). All the procedures in this investigation were performed by one orthopedic surgeon (HM) using a lowcontact-stress mobile-bearing Universal rotational platform (Depuy Orthopaedics Inc., Kobe city, Japan) and a medial parapatellar approach. The inclusion criteria consisted of (i) medial $\mathrm{OA}$ and (ii) more than $120^{\circ}$ in preoperative knee flexion ROM, because the preoperative ROM and softtissue condition affect postoperative $\mathrm{ROM}^{8-10)}$. The exclusion criteria consisted of (i) neurological conditions such as Parkinson disease or stroke, (ii) severe symptoms in other lower extremity joints, and (iii) an inability to participate in a follow-up period of up to 12 months after TKA. The ethics committee of Anshin hospital approved all the procedures performed in the present study (Assignment number. 60 ), and all patients provided written informed consent in accordance with the declaration of Helsinki before participating.

\section{Rehabilitation protocol}

The rehabilitation protocol consisted of 6 days of inpatient rehabilitation and once-a-week outpatient rehabilitation for 12 weeks. During the postoperative stay in the hospital, all patients were treated twice daily for 40 minutes by physical therapists. Inpatient rehabilitation consisted of passive knee ROM exercises; patellofemoral joint mobilization as needed; incision mobility; lower extremity flexibility exercises for the quadriceps, calves, and hamstrings; icing; gait training; and functional training for going up and down on stairs. After being discharged from the hospital, patients received outpatient physical therapy once a week for 12 weeks. Outpatient rehabilitation consisted of passive and active knee ROM exercises, quadriceps strengthening, gait training, and activities of daily living training (i.e., climbing and descending stairs). Additionally, all the patients were prescribed a standard home exercise program to be performed twice daily. The home exercise program included active knee ROM exercises and strengthening exercises for the quadriceps, hip abductors, and hip extensors in both weight-bearing and non-weight-bearing conditions. After 12 weeks, patients completed the outpatient rehabilitation and home exercise program.

\section{Assessment \\ 3-1. Knee flexion ROM}

Knee flexion ROM was measured preoperatively and at 5 days, 1 month, and 12 months postoperatively, because our patients were discharged 5 days postoperatively, and the average length of hospital stay in Japan is 1 month postoperatively ${ }^{17,18)}$. Measurement of knee flexion ROM was followed the methods recommended by the Japanese Orthopedic Association and the Japanese Association of Rehabilitation Medicine. Passive ROM of the involved limb was measured every 5 degrees in the supine position. Seven trained physical therapists carried out all measurements. The reliability of knee flexion goniometric measurement after TKA is appropriate in the acute (intraclass correlation coefficient [ICC], 0.89) and outpatient settings (ICC, 0.81$0.87)^{19,20)}$. Therefore, we measured knee flexion ROM using a goniometer.

\section{3-2. Walking Pain}

Walking pain is the quantification of knee pain just after walking at a normal speed at 5 days and 1 month postoperatively. The numeric rating scale (NRS) is a valid and reliable instrument used in clinical practice due to its high degree of sensitivity ${ }^{21)}$. In the present study, NRS was used to quantify knee pain in the surgical side during walking. Patients were asked to verbally rate the walking pain on a scale from 0 to 10 immediately after walking, with 0 representing no pain and 10 representing the worst pain imaginable.

\section{Statistical analysis}

Continuous variables are expressed as mean \pm standard deviation of the mean (SD), and ordinal and categorical variables are expressed as number $(\%)$. The goal of knee flexion ROM at 12 months after TKA was set at $120^{\circ}$ because this is sufficient for most activities of daily living, 
Table 1. Demographic data of patients in the acheivement and no-acheivement groups

\begin{tabular}{|c|c|c|c|c|}
\hline Variables & $\begin{array}{c}\text { all } \\
(\mathrm{n}=193)\end{array}$ & $\begin{array}{l}\text { achievement group } \\
(\mathrm{n}=152)\end{array}$ & $\begin{array}{l}\text { no-achievement group } \\
\qquad(\mathrm{n}=41)\end{array}$ & $p$-value \\
\hline Gender: female/male, n (\%) & $144 / 49(76 / 24)$ & $115 / 37(77 / 23)$ & $30 / 11(75 / 25)$ & 0.68 \\
\hline Age, yrs & $73.2 \pm 7.7$ & $73.1 \pm 7.8$ & $73.7 \pm 7.4$ & 0.66 \\
\hline Height, $\mathrm{cm}$ & $153.5 \pm 7.7$ & $154.0 \pm 7.4$ & $151.9 \pm 8.6$ & 0.13 \\
\hline Weight, kg & $60.5 \pm 10.2$ & $60.2 \pm 10.0$ & $61.8 \pm 10.8$ & 0.38 \\
\hline $\mathrm{BMI}^{\mathrm{a}}, \mathrm{kg} / \mathrm{m}^{2}$ & $25.8 \pm 4.1$ & $25.5 \pm 4.3$ & $26.6 \pm 3.1$ & 0.12 \\
\hline surgical time, time & $99.3 \pm 10.9$ & $99.6 \pm 11.0$ & $98.2 \pm 10.0$ & 0.47 \\
\hline Preoperative FTA ${ }^{\mathrm{b}}$, degree & $181.3 \pm 4.7$ & $181.6 \pm 4.7$ & $180.2 \pm 4.3$ & 0.12 \\
\hline $\mathrm{KL}^{\mathrm{c}}$ grade in the involved limb, $\mathrm{n}(\%)$ & & & & 0.55 \\
\hline Grade3 & $19(9.8)$ & $16(10.5)$ & $3(7.3)$ & \\
\hline Grade4 & $174(90.2)$ & $136(89.5)$ & $38(92.7)$ & \\
\hline walking pain at postoperative 5 days, score & $2.5 \pm 1.8$ & $2.4 \pm 1.7$ & $2.9 \pm 1.9$ & 0.16 \\
\hline walking pain at postoperative 1 month, score & $2.4 \pm 1.8$ & $2.3 \pm 1.7$ & $4.0 \pm 1.6$ & $<0.01$ \\
\hline \multicolumn{5}{|l|}{ knee flexion ROM, degree } \\
\hline Preoperatively & $124.7 \pm 4.9$ & $125.4 \pm 5.1$ & $122.1 \pm 3.1$ & 0.42 \\
\hline 5 days postoperatively & $92.7 \pm 6.7$ & $94.0 \pm 6.8$ & $90.4 \pm 6.0$ & $<0.01$ \\
\hline 1 month postoperatively & $111.2 \pm 10.4$ & $113.4 \pm 9.2$ & $103.0 \pm 10.6$ & $<0.01$ \\
\hline 12 months postoperatively & $121.1 \pm 8.9$ & $124.5 \pm 5.0$ & $108.5 \pm 9.2$ & $<0.01$ \\
\hline
\end{tabular}

aBMI; body mass index, bFTA; femorotibial angle,

cKL grade; Kellgren-Lawrence grade of knee osteoarthritis

Continuous variables were expressed as mean $\pm \mathrm{SD}$, and ordinal and categorical variables were expressed as number $(\%)$

including kneeling, and is performed with less risk to the patellofemoral joint ${ }^{22,23}$.

Initially, the characteristics were compared between participants who met the goal (achievement group) versus those who did not (no-achievement group). The parametric values were compared using the unpaired $t$-test, while the non-parametric values were compared using the MannWhitney U test.

Thereafter, single and multiple logistic-regression analyses were performed to investigate the relationship between knee flexion ROM at 5 days or 1 month postoperatively, and at 12 months. In addition, we performed the logistic-regression analysis investigating the relationship between that at preoperatively with that at 12 months and compared it with the results at 5 days and 1 month postoperatively. The dependent variable was the goal of $120^{\circ}$ in knee flexion ROM at 12 months (0: no-achievement group, 1: achievement group). The independent variables were knee flexion ROM at 5 days or that at 1 month postoperatively, separately. Age, gender (0: male, 1: female), BMI, and walking pain at 5 days or 1 month were considered potential confounders.

Finally, we identified the cut-off point for the degree of knee flexion ROM at 5 days and 1 month postoperatively for achieving $120^{\circ}$ of knee flexion ROM at 12 months using receiver operating curve analysis with the Youden index. We also calculated the cut-off point for the degree of knee flexion ROM at preoperatively and compared it with that at 5 days and 1 month postoperatively.
Statistical significance was accepted at $p<0.05$. All analyses were carried out using SPSS for Windows 21.0.0 version (IBM Corp., Tokyo, Japan).

\section{Results}

Table 1 summarizes the gender, age, height, weight, BMI, surgical time, preoperative femorotibial angle, knee OA grade of $\mathrm{K} / \mathrm{L}$ grade, and walking pain at 5 days and 1 month postoperatively for each patient. Patients with more than $120^{\circ}$ of knee flexion ROM at 12 months after TKA included 152 of 193 patients (78.7\%), while 41 of 193 patients $(21.3 \%)$ had less than $120^{\circ}$ of ROM. There were no significant differences in the characteristics such as age, BMI, duration of surgery, and grade of OA in the involved limb between the two groups at baseline. Walking pain score at 1 month was higher in the no-achievement group than in the achievement group $(4.0 \pm 1.6$ vs. $2.3 \pm 1.7, \mathrm{p}<$ $0.01)$.

Table 2 summarizes the results of the logistic regression analysis to investigate the effect of knee flexion ROM at 5 days or 1 month on the ROM at 12 months. Knee flexion ROM at 5 days and 1 month were significantly associated with achievement of knee flexion ROM at 12 months (knee flexion ROM at 5 days; odds ratio $(\mathrm{OR})=1.10$ [95\% confidence interval $(\mathrm{CI}), 1.03-1.20], \mathrm{p}=0.01$, HosmerLemeshow test $p=0.27$, knee flexion ROM at 1 month; $\mathrm{OR}=1.13[95 \%$ CI, 1.08-1.18], $\mathrm{p}<0.01$, HosmerLemeshow test, $\mathrm{p}=0.81$ ) adjusting for age, gender, body 
Table 2. Results of logistic legression analysis to investigate the effect of knee flexion ROM at 5 days or 1 month on the acheivement of knee flexion ROM at 12 months.

\begin{tabular}{lcrrr}
\hline \multirow{2}{*}{ Independent variables } & \multicolumn{2}{c}{ Crude model } & \multicolumn{2}{c}{ Adjusted model } \\
\cline { 2 - 5 } & OR (95\% CI) & $p$ value & OR (95\% CI) & $p$ value \\
\hline Knee flexion ROM at 5 days after TKA & $1.09(1.02-1.16)$ & 0.02 & $1.10(1.03-1.20)$ & 0.01 \\
Age & & & $0.97(0.92-1.02)$ & 0.20 \\
Gender & & $0.58(0.25-1.32)$ & 0.19 \\
Body mass index & & $0.94(0.86-1.03)$ & 0.17 \\
Walking pain at 5 days & $1.12(1.07-1.16)$ & $<0.01$ & $0.99(0.80-1.22)$ & 0.89 \\
Knee flexion ROM at 1 month after TKA & & $0.94(0.88-0.99)$ & 0.03 \\
Age & & & $0.40(0.15-1.10)$ & 0.08 \\
Gender & & $0.98(0.89-1.08)$ & 0.63 \\
Body mass index & & $0.86(0.67-1.09)$ & 0.22 \\
Walking pain at 1 month & & & $1.23(1.10-1.37)$ & $<0.01$ \\
Knee flexion ROM at preoperatively & $1.22(1.09-1.35)$ & $<0.01$ & $0.99(0.94-1.04)$ & 0.61 \\
Age & & $0.59(0.25-1.37)$ & 0.22 \\
Gender & & $0.92(0.84-1.02)$ & 0.07 \\
Body mass index & & $1.16(0.99-1.36)$ & 0.06 \\
Walking pain at preoperatively & & & & \\
\hline
\end{tabular}

Dependent variable: the outcome of the achievement of knee flexion ROM at 12 months.

Independent variable: knee flexion ROM at 5 days and 1 month after TKA separately

Age, gender, body mass index and walking pain at 5 days or 1 month were considered potential confounders.

OR; odds ratio, $\mathrm{CI}$; confidence interval

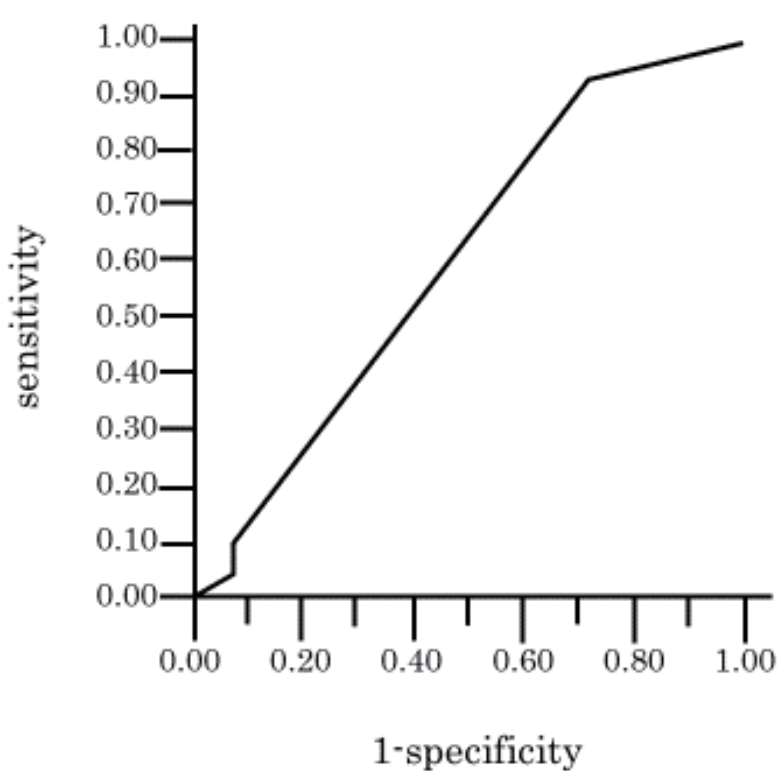

Figure 1. Receiver operating characteristic curve analysis at 5 days after TKA.

mass index, and walking pain. (preoperatively; $\mathrm{OR}=1.23$ [95\% CI, 1.10-1.37], $\mathrm{p}<0.01$, Hosmer-Lemeshow test $\mathrm{p}=$ $0.21)$

The cut-off points of knee flexion ROM for achieving $120^{\circ}$ of knee flexion at 12 month were $85^{\circ}$ for knee flexion ROM at 5 days postoperatively [sensitivity: 0.27 , specificity: 0.94, AUC: 0.63] (Figure 1), and $105^{\circ}$ for 1 month

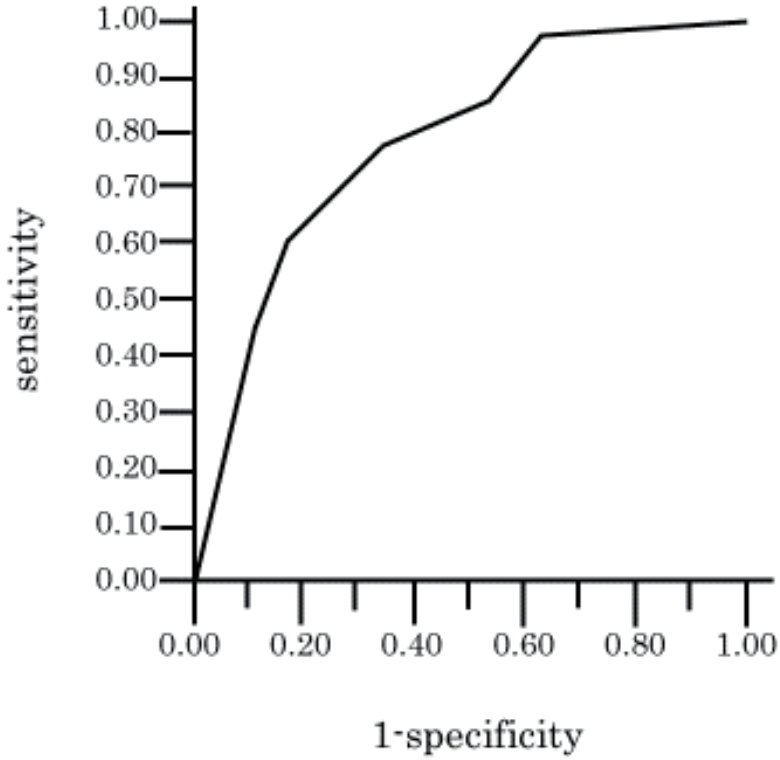

Figure 2. Receiver operating characteristic curve analysis at 1 month after TKA.

postoperatively [sensitivity: 0.63 , specificity: 0.82, AUC: 0.80] (Figure 2). (The cut-off point was $122^{\circ}$ for knee flexion ROM preoperatively [sensitivity: 0.68 , specificity: 0.61 , area under curve (AUC): 0.68]). 


\section{Discussion}

Our study aimed to investigate whether knee flexion ROM during the acute phases of postoperative rehabilitation were useful for predicting achievement of knee flexion ROM at 12 months postoperatively, and to clarify the degree of knee flexion ROM during the acute phases that represents the cut-off point for achieving the goal of knee flexion $\mathrm{ROM}\left(120^{\circ}\right)$ at 12 months after TKA. The primary results of the present study were that knee flexion ROM at 1 month was a more useful parameter than that at preoperatively and 5 days postoperatively for predicting the achievement of knee flexion ROM at 12 months. Moreover, the cut-off value at 1 month after TKA for indicating the achievement of $120^{\circ} \mathrm{ROM}$ by 12 months was $105^{\circ}$. To the best of our knowledge, our study is the first to identify the cut-off point and predictive precision for achieving $120^{\circ}$ of knee flexion ROM at 12 months after TKA.

Based on the results of the logistic-regression analyses, we found that knee flexion ROM during the acute postoperative phases was predictive of the achievement of the clinical goal $\left(120^{\circ} \mathrm{ROM}\right)$ at 12 months after TKA. This finding was consistent with the results of a previous study, which demonstrated that knee flexion ROM during the acute phase could predict knee flexion ROM at 2 months postoperatively $^{14)}$. Our findings suggest that if knee flexion ROM is improved within the acute phase in an inpatient or outpatient setting, this may translate to improved long-term outcomes.

We also identified the degree and predictive precision of knee flexion ROM during the acute phase. The predictive precision at 5 days postoperatively showed high specificity, which indicated a high probability that patients will not reach $120^{\circ}$ at 12 months if they fail to reach $85^{\circ}$ of knee flexion ROM at 5 days after TKA. However, the sensitivity and AUC suggest low accuracy. Knee flexion ROM during hospitalization depends on pain, pain tolerance, type of anesthesia used in TKA, and postoperative dosing of pain medications ${ }^{24,25)}$, which may cause a decrease in the predictive precision of knee flexion ROM at this time point. Due to these reasons, the sensitivity and AUC decreased, indicating a reduced accuracy. Therefore, we considered that the cut-off ROM at 5 days postoperatively might not be useful in predicting the achievement of knee flexion ROM at 12 months after TKA.

Conversely, all the constituents of predictive accuracy at 1 month were higher than that at 5 days. We found that walking pain at 1 month after TKA in the no-achievement group was higher than that in the achievement group, suggesting that pain during walking could affect knee flexion ROM at 1 month after TKA. Muscle spasm of the knee joint, owing to postoperative pain, led to a stiff knee during walking $^{26)}$. As the patients with stiff knee gait showed decreased knee flexion ROM during walking, these gait pat- terns might have affected knee flexion ROM. Furthermore, our results showed the predictive accuracy at 1 month postoperatively was higher than that preoperatively. Bade MJ et al. reported that preoperative knee flexion ROM can predict long-term knee flexion ROM than postoperative knee flexion ROM at 2 days $^{27}$. Our results indicate that it is inadequate to predict long-term ROM based on preoperative ROM, and it is essential to check ROM carefully until 1 month postoperatively.

These results suggest that it is essential to restore knee flexion ROM within 1 month to reach the critical flexion ROM at 12 months. The results from the present investigation may be useful for establishing a rehabilitation plan and procedure to identify patients at high risk of poor knee flexion ROM at 12 months. In addition, our results indicate that establishing clear goals of knee flexion ROM during the acute phases following surgery may provide reassurance to the patients with regards to the increased chance of good ROM at 12 months after TKA.

This study has several methodological limitations. First, to remove the influence of preoperative knee ROM, patients with less than $120^{\circ}$ of preoperative knee flexion ROM were excluded as $120^{\circ}$ was set as the clinical goal of knee flexion ROM at 12 months. Second, we did not examine potential confounders such as knee extensor strength, surgical variables, comorbidities, and psychological factors that might have affected knee flexion ROM after TKA ${ }^{28-31}$. Finally, we did not confirm whether home exercise was performed after 3 months postoperatively. This might have affected the results of this study. Given these limitations, care should be taken when extrapolating the results of this investigation to all individuals who undergo TKA.

\section{Conclusions}

Knee flexion ROM during the acute postoperative phases is useful in predicting the achievement of knee flexion ROM at 12 months in patients who undergo TKA. Furthermore, we identified the cut-off ROM and predictive precision of ROM values measured during the acute postoperative phase. Our findings highlight the importance of early improvement in knee flexion ROM after TKA, and demonstrate that ROM at 1 month postoperatively indicates the likelihood of achieving the clinical goal of knee flexion ROM at 12 months after TKA.

Acknowledgements: We acknowledge all subjects and all staff members who participated in this study.

Conflict of Interest: None.

\section{References}

1) Ha CW, Park YB, et al.: Increased range of motion is important for functional outcome and satisfaction after total knee arthro- 
plasty in Asian patients. J Arthroplasty. 2016; 31: 1199-1203.

2) Ritter MA, Lutgring JD, et al.: The role of flexion contracture on outcomes in primary total knee arthroplasty. J Arthroplasty. 2007; 22: 1092-1096.

3) Miner AL, Lingard EA, et al.; Kinemax Outcomes Group: Knee range of motion after total knee arthroplasty: how important is this as an outcome measure? J Arthroplasty. 2003; 18: 286-294.

4) Padua R, Ceccarelli E, et al.: Range of motion correlates with patient perception of TKA outcome. Clin Orthop Relat Res. 2007; 460: 174-177.

5) Rowe PJ, Myles CM, et al.: The effect of total knee arthroplasty on joint movement during functional activities and joint range of motion with particular regard to higher flexion users. J Orthop Surg (Hong Kong). 2005; 13: 131-138.

6) Devers BN, Conditt MA, et al: Does greater knee flexion increase patient function and satisfaction after total knee arthroplasty? J Arthroplasty. 2011; 26: 178-186.

7) Wimmer MA, Nechtow W, et al.: Knee flexion and daily activities in patients following total knee replacement: a comparison with ISO standard 14243. Biomed Res Int. 2015 Aug 11. doi: $10.1155 / 2015 / 157541$

8) Farahini H, Moghtadaei M, et al.: Factors influencing range of motion after total knee arthroplasty. Iran Red Crescent Med J. 2012; 14: 417-421.

9) Ryu J, Saito S, et al.: Factors influencing the postoperative range of motion in total knee arthroplasty. Bull Hosp Jt Dis. 1993; 53: 35-40.

10) Ritter MA, Harty LD, et al.: Predicting range of motion after total knee arthroplasty. Clustering, log-linear regression, and regression tree analysis. J Bone Joint Surg Am. 2003; 85-A: 12781285 .

11) Gatha NM, Clarke HD, et al.: Factors affecting postoperative range of motion after total knee arthroplasty. J Knee Surg. 2004; 17: 196-202.

12) Schurman DJ, Matityahu A, et al.: Prediction of postoperative knee flexion in Insall-Burstein II total knee arthroplasty. Clin Orthop Relat Res. 1998; 353: 175-184

13) Geijsen GJ, Heesterbeek PJ, et al.: Do tibiofemoral contact point and posterior condylar offset influence outcome and range of motion in a mobile-bearing total knee arthroplasty? Knee Surg Sports Traumatol Arthrosc. 2014; 22: 550-555.

14) Naylor JM, Ko V, et al.: Is discharge knee range of motion a useful and relevant clinical indicator after total knee replacement? Part 2. J Eval Clin Pract. 2012; 18: 652-658.

15) Ebert JR, Munsie C, et al.: Guidelines for the early restoration of active knee flexion after total knee arthroplasty: implications for rehabilitation and early intervention. Arch Phys Med Rehabil. 2014; 95: 1135-1140.

16) Zhou Z, Yew KS, et al.: Recovery in knee range of motion reaches a plateau by 12 months after total knee arthroplasty.
Knee Surg Sports Traumatol Arthrosc. 2015; 23: 1729-1733.

17) Ishii $Y$, Noguchi H, et al.: Length of hospital stay with patientdependent determination in bilateral scheduled staged total knee arthroplasty. Eur J Orthop Surg Traumatol. 2014; 24: 961-965.

18) Yasunaga $\mathrm{H}$, Tsuchiya $\mathrm{K}$, et al.: Analysis of factors affecting operating time, postoperative complications, and length of stay for total knee arthroplasty: nationwide web-based survey. J Orthop Sci. 2009; 14: 10-16.

19) Lenssen AF, van Dam EM, et al.: Reproducibility of goniometric measurement of the knee in the in-hospital phase following total knee arthroplasty. BMC Musculoskelet Disord. 2007 Aug 7; 8. doi: 10.1186/1471-2474-8-83. PubMed PMID: 17705860; PubMed Central PMCID: PMC2040146.

20) Jakobsen TL, Christensen M, et al.: Reliability of knee joint range of motion and circumference measurements after total knee arthroplasty: does tester experience matter? Physiother Res Int. 2010; 15: 126-134.

21) Williamson A and Hoqqart B: Pain: a review of three commonly used pain rating scales. J Clin Nurs. 2005; 14: 798-804.

22) Rowe PJ, Myles CM, et al:: Knee joint kinematics in gait and other functional activities measured using flexible electrogoniometry: how much knee motion is sufficient for normal daily life? Gait Posture. 2000; 12: 143-155.

23) Lee TQ: Biomechanics of hyperflexion and kneeling before and after total knee arthroplasty. Clin Orthop Surg. 2014; 6: 117126.

24) Chan EY, Blyth FM, et al.: Acute postoperative pain following hospital discharge after total knee arthroplasty. Osteoarthritis Cartilage. 2013; 21: 1257-1263.

25) Dunbar MJ: Subjective outcomes after knee arthroplasty. Acta Orthop Scand Suppl. 2001; 72: 1-63.

26) Levinger P, Menz HB, et al.: Knee biomechanics early after knee replacement surgery predict abnormal gait patterns 12 months postoperatively. J Orthop Res. 2012; 30: 371-376.

27) Bade MJ, Kittelson JM, et al.: Predicting functional performance and range of motion outcomes after total knee arthroplasty. Am J Phys Med Rehabili. 2014; 93: 579-585.

28) Wada O, Nagai K, et al.: Diabetes is a risk factor for restricted range of motion and poor clinical outcome after total knee arthroplasty. J Arthroplasty. 2016; 31: 1933-1937.

29) Forsythe ME, Dunbar MJ, et al: : Prospective relation between catastrophizing and residual pain following knee arthroplasty: two-year follow-up. Pain Res Manag. 2008; 13: 335-341.

30) Hirakawa Y, Hara M, et al.: The relationship among psychological factors, neglect-like symptoms and postoperative pain after total knee arthroplasty. Pain Res Manag. 2014; 19: 251-256.

31) Rama KR, Apsingi $S$, et al.: Timing of tourniquet release in knee arthroplasty. Meta-analysis of randomized, controlled trials. J Bone Joint Surg Am. 2007; 89: 699-705. 